\title{
Lung Shunt Reduction for Yttrium-90 Radioembolization: Chemoembolization Versus Radioembolization
}

\author{
HYO-CHEOL KIM ${ }^{1}$, JIN WOO CHOI ${ }^{1}$, MYUNGSU LEE ${ }^{1}$, YOON JUN KIM ${ }^{2}$, \\ JIN CHUL PAENG ${ }^{3}$ and JIN WOOK CHUNG ${ }^{1}$ \\ ${ }^{1}$ Department of Radiology, Seoul National University Hospital, \\ Seoul National University College of Medicine, Seoul, Republic of Korea; \\ ${ }^{2}$ Department of Internal Medicine, Seoul National University Hospital, \\ Seoul National University College of Medicine, Seoul, Republic of Korea; \\ ${ }^{3}$ Department of Nuclear Medicine, Seoul National University Hospital, \\ Seoul National University College of Medicine, Seoul, Republic of Korea
}

\begin{abstract}
Aim: To evaluate the efficacy of chemoembolization versus radioembolization in reducing lung shunt fraction (LSF) in patients with hepatocellular carcinoma (HCC). Patients and Methods: In this retrospective study, from March 2012 to January 2021, 457 patients with HCC underwent planning angiography and ${ }^{99 m}$ Tc-macroaggregated albumin imaging for possible yttrium-90 radioembolization. Ten patients underwent radioembolization, and seven patients underwent conventional chemoembolization for LSF reduction, and a second ${ }^{99 m}$ Tc-macroaggregated albumin imaging was obtained approximately 1 month later. LSF under both procedures was compared with the Mann-Whitney U-test and the Wilcoxon signed-rank test. Results: In the radioembolization group, the mean first and second LSF were $13.0 \pm 6.9 \%$ and $20.9 \pm 9.6 \%$, respectively $(p=0.059)$; after radioembolization, LSF was lower in three patients but higher in seven patients. In the chemoembolization group, the mean first and second LSF were $26.1 \pm 17.3 \%$ and $8.7 \pm 5.5 \%$, respectively ( $p=0.018)$; after chemoembolization, LSF was reduced in all seven patients. Conclusion: Chemoembolization appears to be more effective in reducing LSF within 1 month compared with radioembolization.
\end{abstract}

This article is freely accessible online.

Correspondence to: Hyo-Cheol Kim, MD, Department of Radiology, Seoul National University Hospital, \#101 Daehak-ro, Jongno-gu, Seoul, 03080, Republic of Korea. Tel: +82 220722584, Fax: +82 27436385, e-mail: angiointervention@gmail.com

Key Words: Hepatocellular carcinoma, radioembolization, lung shunt reduction.
Radioembolization with yttrium-90 microspheres is a potent form of transarterial therapy for patients with hepatocellular carcinoma (HCC), with minimal post-embolization syndrome (1). Boosted radioembolization (with a mean absorbed dose to the target tissue $>150 \mathrm{~Gy}$ ) has been found to prolong the survival of patients with good liver function (2). The recent LEGACY study demonstrated excellent tumor response in patients with solitary $\mathrm{HCC}(\leq 8 \mathrm{~cm})$, with a median absorbed dose to the target tissue of 410 Gy (3). Unlike chemoembolization, however, radioembolization cannot be performed in patients with a high lung shunt fraction (LSF) because of possible radiation-induced pneumonitis (4). Using glass microspheres, a total lung dose of $30 \mathrm{~Gy}$ per treatment, or $50 \mathrm{~Gy}$ in a lifetime, is considered the upper limit (4). The lung dose depends on the absolute LSF as well as the target tissue volume and desired mean absorbed dose to the target tissue. For example, when LSF is $10 \%$ and the target volume is $1,800 \mathrm{ml}$, boosted radioembolization is not an option because the lung dose would be over 30 Gy with a mean absorbed dose to the target tissue of $150 \mathrm{~Gy}$.

When the estimated lung dose is over $30 \mathrm{~Gy}$, radioembolization may be tried after an LSF reduction procedure; alternatively, another treatment modality (chemoembolization, surgical resection, or systemic therapy) may be an option. Several methods can reduce LSF, including bland embolization, chemoembolization, low-dose radioembolization, hepatic vein occlusion with a balloon catheter, and systemic chemotherapy (5-11). At the Authors' Institute, chemoembolization or radioembolization is commonly recommended for LSF reduction in patients with a high estimated lung dose. The purpose of this study was to evaluate the efficacy of chemoembolization versus radioembolization for LSF reduction in patients with HCC. 


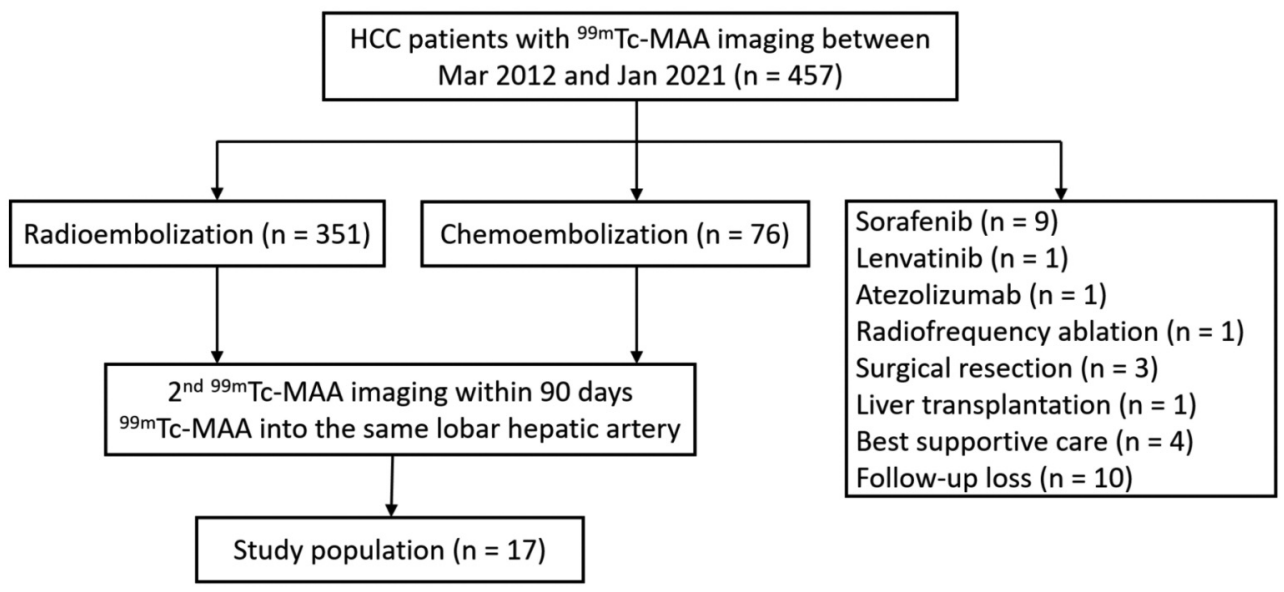

Figure 1. Flowchart of the study population. MAA: Macroaggregated albumin.

\section{Patients and Methods}

Patients. This single-center, retrospective study was approved by the Institutional Review Board (number 2101-165-1191), and informed consent was waived. From March 2012 to January 2021, 457 patients with HCC underwent planning angiography and $99 \mathrm{mTc}-$ macroaggregated albumin (MAA) imaging for possible yttrium-90 radioembolization. Three hundred and fifty-one patients underwent radioembolization, and 106 patients were managed with chemoembolization $(n=76)$ or other treatment $(n=30)$ (Figure 1).

The inclusion criteria were as follows: i) Chemoembolization or radioembolization performed for LSF reduction; ii) a second $99 \mathrm{mTc}-$ MAA imaging performed within 90 days of the first treatment for the primary target tumor; and iii) the patient received a $99 \mathrm{mTc}-\mathrm{MAA}$ injection into the same lobar hepatic artery for the first and second 99mTc-MAA imaging. Exclusion criteria were i) Previous chemoembolization, ablation therapy, or systemic therapy for the primary target tumor; and ii) repeated ${ }^{99} \mathrm{~m}$ Tc-MAA imaging for bi-

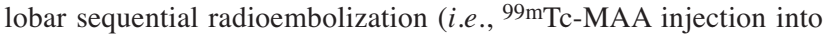
the contralateral lobar hepatic artery). Among the 457 patients, 10 underwent radioembolization, and seven patients underwent conventional chemoembolization for LSF reduction, after which a second 99mTc-MAA imaging was obtained (Figure 1).

Seventeen male patients (mean age: $59.9 \pm 13.4$ years; age range: $37-$ 83 years) therefore comprised the study population. The demographic characteristics of the patients are summarized in Table I. The mean primary tumor size was $12.6 \pm 2.4 \mathrm{~cm}$ (range=7.4-18.6 cm). Nine patients $(53 \%)$ had vascular invasion (portal vein/hepatic vein).

Yttrium-90 radioembolization. The detailed protocol for radioembolization has been described in previous studies (12-15). Two interventional radiologists (H.C.K., with 15 years of experience in interventional oncology and M.L. with 10 years of experience) performed all radioembolization procedures. In the early study period (March 2012 to June 2016), radioembolization was performed with standard dosimetry of TheraSphere or SIR-Spheres as recommended by the manufacturers. In the late study period (July 2016 to January 2021), boosted radioembolization (240-360 Gy mean absorbed dose to the target tissue) was used when applicable (i.e., when the estimated lung dose was less than $30 \mathrm{~Gy}$, and liver function was preserved). Low-dose, regular, and boosted radioembolization were defined as $<80 \mathrm{~Gy}, 80-150 \mathrm{~Gy}$, and $>150$ Gy mean absorbed dose to the target tissue, respectively.

Chemoembolization. The detailed protocol for chemoembolization has been described in previous studies (16-18). Under this protocol, an iodized oil emulsion (a mixture of doxorubicin and iodized oil) is injected until the peritumoral portal vein is filled with iodized oil, followed by embolization with gelatin sponge particles. In large HCCs $(>7 \mathrm{~cm})$, an alternative injection of iodized oil emulsion and polyvinyl alcohol particles $(45-150 \mu \mathrm{m})$ is given through the main tumor-feeding branches until near-stasis of the target vessel is achieved, then gelatin sponge particles are added to achieve complete stasis. In all patients, the maximum doses of iodized oil and doxorubicin were $10 \mathrm{ml}$ and $50 \mathrm{mg}$, respectively. In patients with vascular invasion, an additional infusion of cisplatin (50-70 $\mathrm{mg}$ ) was performed after conventional chemoembolization $(17,18)$.

Lung shunt reduction. Between March 2012 and December 2014, only resin microspheres were available at the Authors' Institute. When LSF was greater than $20 \%$, chemoembolization was recommended as a method of LSF reduction. A second planning angiography and a second ${ }^{99}$ Tc-MAA imaging after chemoembolization were performed for only one patient (Figure 2A).

Between January 2015 and June 2016, only glass microspheres were available at the Authors' Institute. When the mean absorbed dose to the target tissue was less than $100 \mathrm{~Gy}$, chemoembolization was recommended as a method of LSF reduction. However, a second planning angiography was not performed for any of the patients treated during this time (Figure 2B).

Between July 2016 and January 2021, both glass and resin microspheres were available, and boosted radioembolization (with a mean absorbed dose to the target tissue dose $>240 \mathrm{~Gy}$ ) was primarily performed. When the mean absorbed dose to the target tissue dose was less than $240 \mathrm{~Gy}$, patients and their referring physicians were given three options: Switching to another treatment modality, undergoing boosted radioembolization after chemoembolization, or undergoing boosted radioembolization after first radioembolization. Sixteen patients underwent a second planning angiography and a second ${ }^{99 \mathrm{~m}}$ Tc-MAA imaging after chemoembolization $(n=6)$ or after 


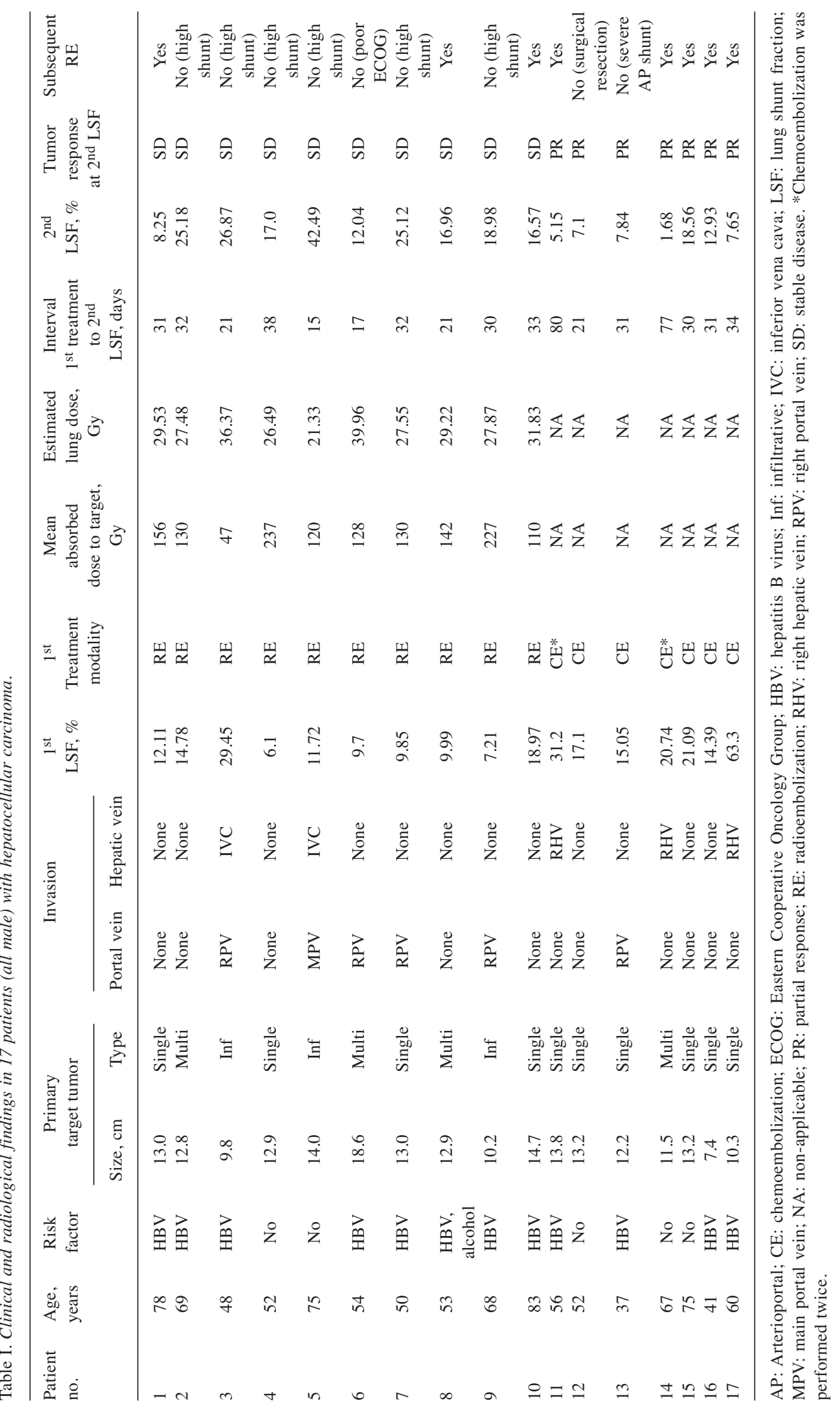


A

HCC patients with ${ }^{99 m} \mathrm{Tc}-\mathrm{MAA}$ imaging between

Mar 2012 and Dec $2014(n=35)$

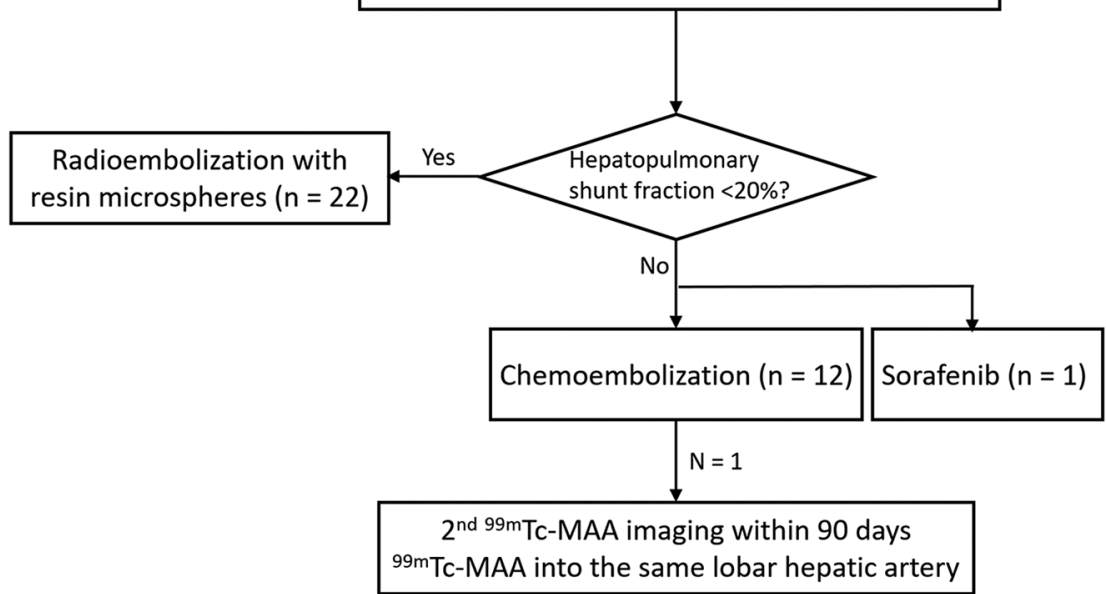

B

\begin{tabular}{|c|}
\hline $\begin{array}{c}\text { HCC patients with } \\
\text { 99mTc-MAA imaging between } \\
\text { Jan } 2015 \text { and Jun } 2016(n=26)\end{array}$ \\
\hline
\end{tabular}
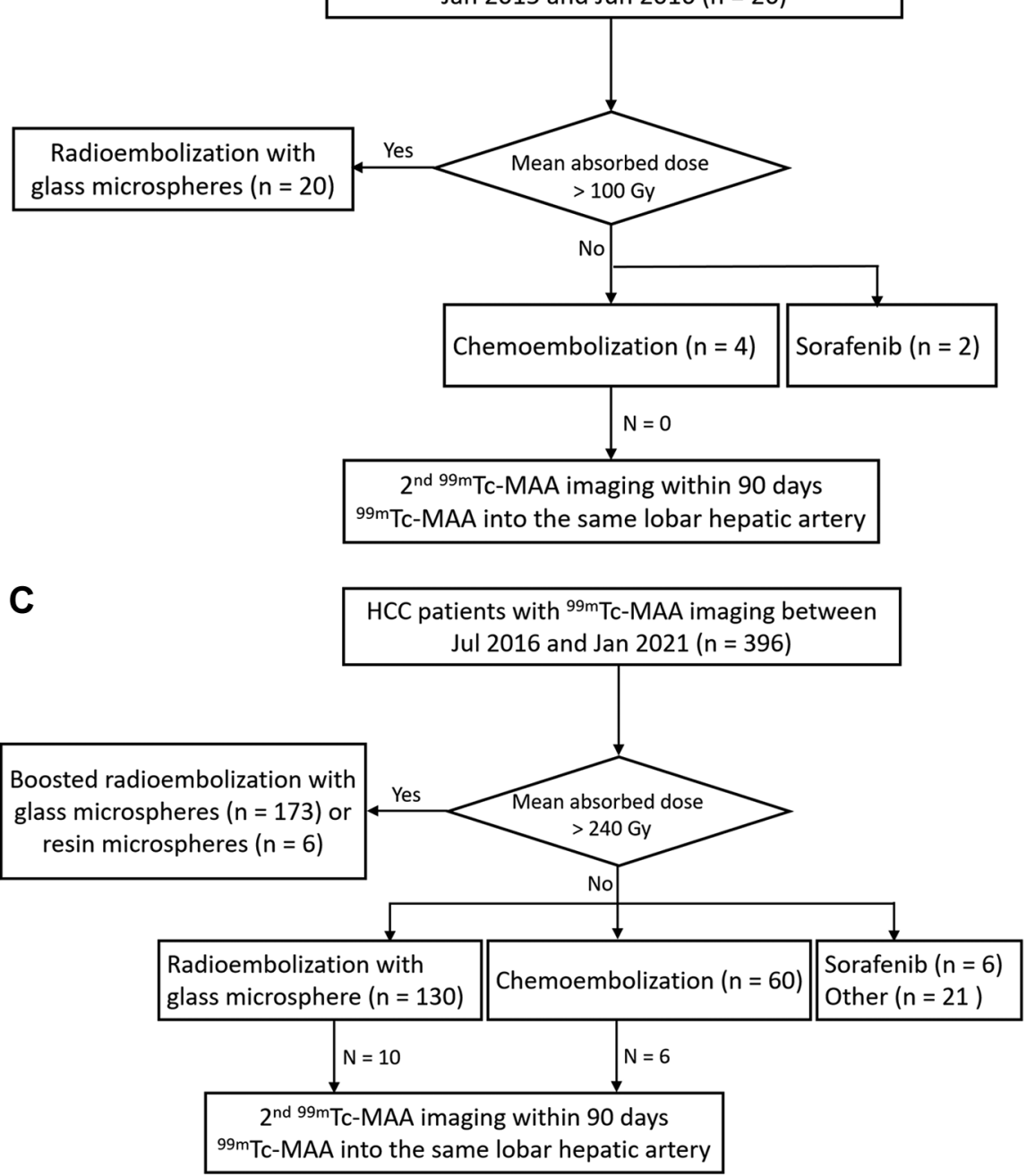

Figure 2. Flowchart of the study population according to the study period. A: Study population between March 2012 and December 2014. B: Study population between January 2015 and June 2016. C: Study population between July 2016 and January 2021. MAA: Macroaggregated albumin. 
A

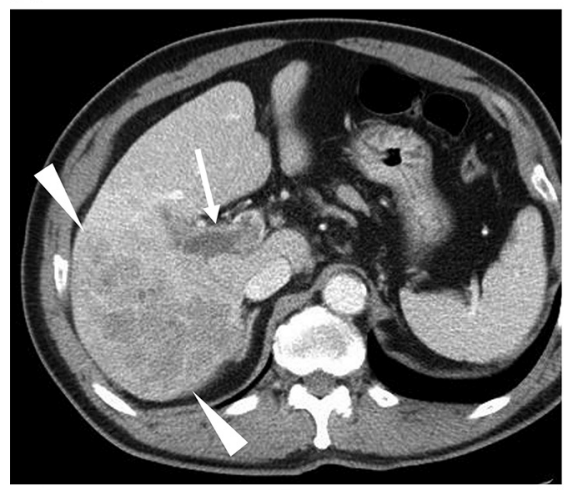

B

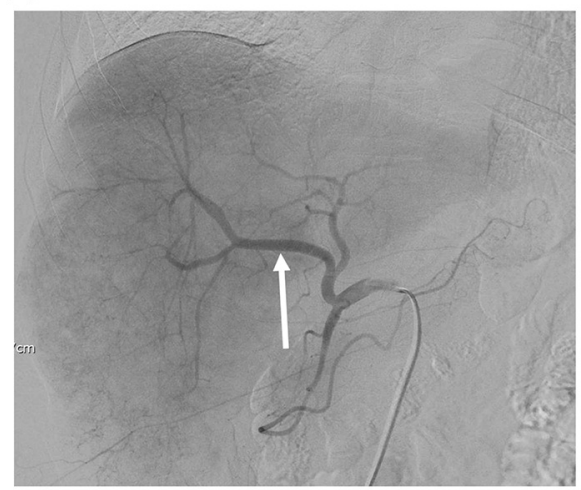

C

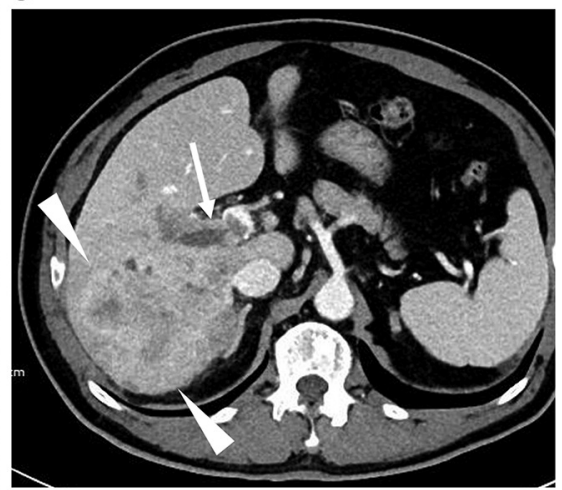

Figure 3. A 68-year-old man (patient number 9) with hepatocellular carcinoma. A: Computed tomography showing ill-defined tumor (arrowheads) in the right lobe of the liver and a tumor thrombus in the right portal vein (arrow). B: Common hepatic arteriogram showing ill-defined hypervascular tumor blush in the right lobe of the liver. ${ }^{99 m}$ Tc-Macroaggregated albumin was injected into the right hepatic artery (arrow), and the lung shunt fraction was $7.21 \%$. Radioembolization was performed via the right hepatic artery; radiation activity at administration was 7.89 GBq, and the estimated lung dose was $27.87 \mathrm{~Gy}$. C: Computed tomography 3 weeks after radioembolization showing stable disease of ill-defined tumor (arrowheads) and portal vein thrombus (arrow). A second macroaggregated albumin imaging was performed 4 weeks after radioembolization, and lung shunt fraction increased to $18.98 \%$.

first radioembolization $(\mathrm{n}=10)$ (Figure $2 \mathrm{C})$. The second planning angiography and second ${ }^{99 \mathrm{~m}} \mathrm{Tc}-\mathrm{MAA}$ imaging were performed approximately 1 month after the LSF procedure.

Patients chose their LSF reduction procedure (chemoembolization or radioembolization) after being informed about the benefits and risks of each. The performance of a second planning angiography and a second ${ }^{99}$ Tc-MAA imaging was determined in consultation with the referring physician and interventional radiologists.

Analysis. Radiological and clinical data were retrospectively collected, and tumor response was evaluated with modified response evaluation criteria in solid tumors (mRECIST) (19). The MannWhitney $U$-test was used to compare LSF between the two groups. The first and second LSF in each group was compared using the Wilcoxon signed-rank test. A $p$-value of less than 0.05 was considered statistically significant. All statistical analyses were performed with SPSS version 25.0 software (IBM, Armonk, NY, USA).

\section{Results}

In the radioembolization group, the mean first and second LSF were $13.0 \pm 6.9 \%$ and $20.9 \pm 9.6 \%$, respectively $(p=0.059)$ (Table II). Only one patient underwent low-dose radioembolization (mean absorbed dose of $47 \mathrm{~Gy}$ ), while nine patients underwent regular $(n=6)$ or boosted $(n=3)$ radioembolization. After radioembolization, LSF was lower in three patients but higher in the remaining seven. All 10 patients showed stable disease at the time of the second planning angiography (Figure 3). Three patients underwent a second session of radioembolization, while seven patients did not.

In the chemoembolization group, the mean first and second LSF were $26.1 \pm 17.3 \%$ and $8.7 \pm 5.5 \%$, respectively
Table II. Statistical analysis of lung shunt fraction (LSF).

\begin{tabular}{lccc}
\hline & $\begin{array}{c}\text { 90Y-Radioembolization } \\
\text { group (n=10) }\end{array}$ & $\begin{array}{c}\text { Chemoembolization } \\
\text { group (n=7) }\end{array}$ & $p$-Value* \\
\hline $1^{\text {st }}$ LSF (\%) & $13.0 \pm 6.9$ & $26.1 \pm 17.3$ & 0.010 \\
$2^{\text {nd }}$ LSF (\%) & $20.9 \pm 9.6$ & $8.7 \pm 5.5$ & 0.005 \\
$p$-Value & 0.059 & 0.018 & \\
\hline
\end{tabular}

Values are the mean $\pm \mathrm{SD}$. *Mann-Whitney $U$-test. "Wilcoxon signedrank test.

( $p=0.018)$ (Table II). LSF after chemoembolization was reduced in all seven patients. All seven patients showed a partial response at the time of the second planning angiography (two underwent two sessions of chemoembolization) (Figure 4). Five patients underwent subsequent radioembolization. One patient was not able to undergo radioembolization due to severe arterioportal shunt caused by portal vein tumor thrombosis, and another patient underwent surgical resection instead of radioembolization based on the referring physician's recommendation.

Whereas the first LSF of the chemoembolization group was higher than that of the radioembolization group $(p=0.01)$, the second LSF was lower $(p=0.005)$ (Table II).

\section{Discussion}

Chemoembolization and bland embolization have been reported to be effective in reducing $\operatorname{LSF}(6,8-10)$, and the results of this study are compatible with those of previous 

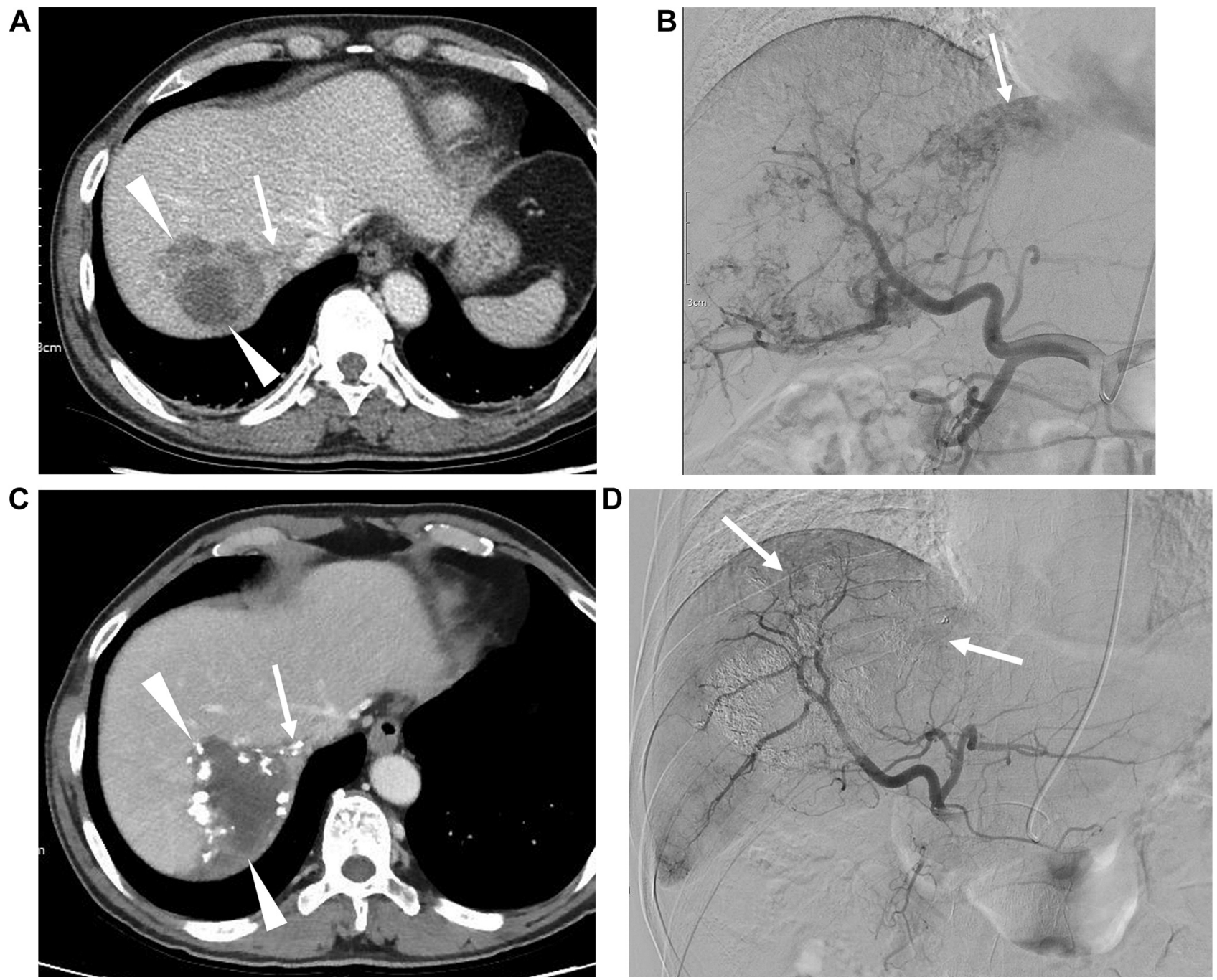

Figure 4. A 60-year-old man (patient number 17) with hepatocellular carcinoma. A: Computed tomography scan showing a large mass (arrowheads) in the right lobe of the liver and a tumor thrombus in the right hepatic vein (arrow). B: Celiac angiogram showing hypervascular tumor blush in the right lobe of the liver and hepatic venous shunting through the right hepatic vein (arrow). ${ }^{99 m}$ Tc-Macroaggregated albumin was injected into the right hepatic artery, and the lung shunt fraction was 63.3\%. Chemoembolization was performed via the right hepatic artery, caudate artery, and right inferior phrenic artery with alternative injection of iodized oil emulsion (10 ml of Lipiodol and $40 \mathrm{mg}$ of doxorubicin) and gelatin sponge particles. Additional infusion of $50 \mathrm{mg}$ of cisplatin was performed via the right hepatic artery. C: Computed tomography 3 weeks after chemoembolization showing abundant tumor necrosis (arrowheads) and iodized oil accumulation in the right hepatic vein thrombus (arrow). D: A second macroaggregated albumin imaging was performed 5 weeks after chemoembolization. A hepatic angiogram showing a small multifocal residual tumor blush (arrows), and the lung shunt fraction decreased to $7.65 \%$.

studies. However, LSF tended to increase after radioembolization in this study. Low-dose radioembolization (an estimated lung dose less than $30 \mathrm{~Gy}$ ) was mentioned in one review article (11) but we found no further case reports or original articles.

Since radioactive microspheres and MAA are a little larger than the diameter of the liver sinusoid, LSF through normal liver would be negligible. In a recent study with a large study population (20), LSF was clinically negligible in patients with United Network for Organ Sharing T1/T2 HCC, and ${ }^{99 m}$ Tc-MAA imaging can be eliminated when segmental injections are planned. It has been reported that a high LSF is associated with a large tumor burden and vascular invasion $(21,22)$. Thus, we speculated that radioactive microspheres and MAA would be able to pass through HCCs with abnormally dilated vessels, and a reduction of tumor mass might be a direct way of reducing LSF in patients with high LSF. 
Chemoembolization and bland embolization exert their antitumor effect by immediate ischemic injury to the tumor, and partial response may be easily observed on imaging 1 month later. Thus, chemoembolization was effective in reducing LSF in this study. On the other hand, radioembolization does not result in ischemic injury, and it may take several months for radioembolization to show a radiological response (23). In this study, all the patients in the radioembolization group showed stable disease at 1month imaging. Thus, radioembolization was not effective in reducing LSF.

After external radiation therapy, the irradiated liver tissue shows variable enhancement changes that are explained by radiation-induced veno-occlusive disease or fibrotic change (24). Histopathological changes after radioembolization have not yet been described in detail in the literature (25). Radioembolization may cause distortion of the hepatic microvascular structure, which may be the reason why LSF increased in seven patients in this study.

This study has some limitations. Firstly, the study population was relatively small, and further studies are required to draw solid conclusions. Secondly, in the radioembolization group, nine patients received regular or boosted radioembolization rather than low-dose radioembolization. In addition, most patients in the radioembolization group had mildly elevated LSF. Thus, there is a possibility that low-dose radioembolization may be effective in reducing LSF in patients with highly elevated LSF. Thirdly, because the first LSF in the chemoembolization group was higher than that of the radioembolization group, it may be that LSF reduction was more easily achieved in the chemoembolization group. Fourthly, the second LSF was measured approximately 1 month after radioembolization in this study. It is not known how long it takes to reduce LSF with radioembolization. If LSF had been measured 3 months after radioembolization, it is possible that it would have been shown to have decreased further. Finally, baseline characteristics such as tumor type, vascular invasion and first LSF were a little different between the two groups. Because of the small study population, the baseline characteristics were not compared with statistical analysis.

In conclusion, chemoembolization appears to be more effective in reducing LSF within 1 month compared with radioembolization.

\section{Conflicts of Interest}

The Authors have no conflicts of interest to disclose in relation to this study.

\section{Authors' Contributions}

Guarantor of integrity of the entire study: Hyo-Cheol Kim. Study concepts and design: Hyo-Cheol Kim. Literature research: Hyo-
Cheol Kim, Myungsu Lee and Jin Chul Paeng. Clinical studies: Hyo-Cheol Kim, Jin Woo Choi, Myungsu Lee, Yoon Jun Kim, Jin Chul Paeng and Jin Wook Chung. Data analysis: Hyo-Cheol Kim, Jin Woo Choi, Myungsu Lee and Jin Chul Paeng. Stastitical analysis: Hyo-Cheol Kim, Myungsu Lee and Yoon Jun Kim. Article preparation: Hyo-Cheol Kim. Article editing: Jin Woo Choi, Myungsu Lee, Yoon Jun Kim, Jin Chul Paeng and Jin Wook Chung.

\section{References}

1 Salem R, Gilbertsen M, Butt Z, Memon K, Vouche M, Hickey R, Baker T, Abecassis MM, Atassi R, Riaz A, Cella D, Burns JL, Ganger D, Benson AB 3rd, Mulcahy MF, Kulik L and Lewandowski R: Increased quality of life among hepatocellular carcinoma patients treated with radioembolization, compared with chemoembolization. Clin Gastroenterol Hepatol 11(10): 13581365.e1, 2013. PMID: 23644386. DOI: 10.1016/j.cgh.2013.04.028

2 Garin E, Rolland Y, Edeline J, Icard N, Lenoir L, Laffont S, Mesbah H, Breton M, Sulpice L, Boudjema K, Rohou T, Raoul JL, Clement B and Boucher E: Personalized dosimetry with intensification using 90Y-loaded glass microsphere radioembolization induces prolonged overall survival in hepatocellular carcinoma patients with portal vein thrombosis. J Nucl Med 56(3): 339-346, 2015. PMID: 25678490. DOI: 10.2967/jnumed.114.145177

3 Salem R, Johnson GE, Kim E, Riaz A, Bishay V, Boucher E, Fowers K, Lewandowski $\mathrm{R}$ and Padia SA: Yttrium-90 radioembolization for the treatment of solitary, unresectable hepatocellular carcinoma: The LEGACY study. Hepatology, 2021. PMID: 33739462. DOI: 10.1002/hep.31819

4 Riaz A, Lewandowski RJ, Kulik LM, Mulcahy MF, Sato KT, Ryu RK, Omary RA and Salem R: Complications following radioembolization with yttrium-90 microspheres: a comprehensive literature review. J Vasc Interv Radiol 20(9): 1121-30; quiz 1131, 2009. PMID: 19640737. DOI: 10.1016/ j.jvir.2009.05.030

5 Bester L and Salem R: Reduction of arteriohepatovenous shunting by temporary balloon occlusion in patients undergoing radioembolization. J Vasc Interv Radiol 18(10): 1310-1314, 2007. PMID: 17911524. DOI: 10.1016/j.jvir.2007.07.003

6 Rose SC and Hoh CK: Hepatopulmonary shunt reduction using chemoembolization to permit yttrium-90 radioembolization. J Vasc Interv Radiol 20(6): 849-851, 2009. PMID: 19465310. DOI: $10.1016 /$ j.jvir.2009.03.010

7 Theysohn JM, Schlaak JF, Müller S, Ertle J, Schlosser TW, Bockisch A and Lauenstein TC: Selective internal radiation therapy of hepatocellular carcinoma: potential hepatopulmonary shunt reduction after sorafenib administration. J Vasc Interv Radiol 23(7): 949-952, 2012. PMID: 22720895. DOI: 10.1016/ j.jvir.2012.04.007

8 Gaba RC and Vanmiddlesworth KA: Chemoembolic hepatopulmonary shunt reduction to allow safe yttrium-90 radioembolization lobectomy of hepatocellular carcinoma. Cardiovasc Intervent Radiol 35(6): 1505-1511, 2012. PMID: 22526098. DOI: 10.1007/s00270-012-0371-7

9 Ward TJ, Tamrazi A, Lam MG, Louie JD, Kao PN, Shah RP, Kadoch MA and Sze DY: Management of high hepatopulmonary shunting in patients undergoing hepatic radioembolization. J Vasc Interv Radiol 26(12): 1751-1760, 2015. PMID: 26525118. DOI: $10.1016 /$ j.jvir.2015.08.027 
10 Kwok N, Irani Z, Sheth R and Arellano RS: Hepatopulmonary shunt reduction with bland embolization for yttrium-90 radioembolization. Diagn Interv Imaging 97(3): 369-370, 2016. PMID: 26780885. DOI: 10.1016/j.diii.2015.12.005

11 Schiro BJ, Amour ES, Harnain C and Gandhi RT: Management of high hepatopulmonary shunts in the setting of Y90 radioembolization. Tech Vasc Interv Radiol 22(2): 58-62, 2019. PMID: 31079711. DOI: 10.1053/j.tvir.2019.02.004

12 Kim HC, Kim YJ, Lee JH, Suh KS and Chung JW: Feasibility of boosted radioembolization for hepatocellular carcinoma larger than $5 \mathrm{~cm}$. J Vasc Interv Radiol 30(1): 1-8, 2019. PMID: 30293734. DOI: 10.1016/j.jvir.2018.07.002

13 Choi JW, Yoo MY, Kim HC, Paeng JC, Kim YJ and Chung JW: Prophylactic temporary occlusion of the cystic artery using a fibered detachable coil during ${ }^{90} \mathrm{Y}$ radioembolization. Cardiovasc Intervent Radiol 40(10): 1624-1630, 2017. PMID: 28500460. DOI: 10.1007/s00270-017-1688-z

14 Kim HC, Kim YJ, Paeng JC and Chung JW: Yttrium-90 radioembolization of the right inferior phrenic artery in 20 patients with hepatocellular carcinoma. J Vasc Interv Radiol 29(4): 556-563, 2018. PMID: 29373246. DOI: 10.1016/ j.jvir.2017.10.010

15 Kim HC, Lee M, Lee JH, Paeng JC, Kim YJ and Chung JW: Combination of $1^{\text {st }}$ and $2^{\text {nd }}$ week dosing of glass yttrium-90 microspheres for superselective radioembolization. In Vivo 34(5): 2763-2768, 2020. PMID: 32871812. DOI: 10.21873/ invivo. 12100

$16 \mathrm{Kim}$ HC, Miyayama S and Chung JW: Selective chemoembolization of caudate lobe hepatocellular carcinoma: anatomy and procedural techniques. Radiographics 39(1): 289302, 2019. PMID: 30620696. DOI: 10.1148/rg.2019180110

17 Choi JW, Kim HC, Lee JH, Yu SJ, Kim YJ, Yoon JH, Jae HJ, Hur S, Lee M and Chung JW: Transarterial chemoembolization of hepatocellular carcinoma with segmental portal vein tumour thrombus. Eur Radiol 27(4): 1448-1458, 2017. PMID: 27516356. DOI: $10.1007 / \mathrm{s} 00330-016-4511-3$

18 Kim HC, Lee JH, Chung JW, Kang B, Yoon JH, Kim YJ, Lee HS, Jae HJ and Park JH: Transarterial chemoembolization with additional cisplatin infusion for hepatocellular carcinoma invading the hepatic vein. J Vasc Interv Radiol 24(2): 274-283, 2013. PMID: 23369561. DOI: 10.1016/j.jvir.2012.11.002
19 Llovet JM and Lencioni R: mRECIST for HCC: Performance and novel refinements. J Hepatol 72(2): 288-306, 2020. PMID: 31954493. DOI: 10.1016/j.jhep.2019.09.026

20 Gabr A, Ranganathan S, Mouli SK, Riaz A, Gates VL, Kulik L, Ganger D, Maddur H, Moore C, Hohlastos E, Katariya N, Caicedo JC, Kalyan A, Lewandowski RJ and Salem R: Streamlining radioembolization in UNOS T1/T2 hepatocellular carcinoma by eliminating lung shunt estimation. J Hepatol 72(6): 1151-1158, 2020. PMID: 32145255. DOI: 10.1016/j.jhep. 2020.02 .024

21 Gaba RC, Zivin SP, Dikopf MS, Parvinian A, Casadaban LC, Lu Y and Bui JT: Characteristics of primary and secondary hepatic malignancies associated with hepatopulmonary shunting. Radiology 271(2): 602-612, 2014. PMID: 24533871. DOI: 10.1148/radiol.14131969

22 Olorunsola OG, Kohi MP, Behr SC, Kolli PK, Taylor AG, Tong RT, LaBerge JM, Kerlan RK and Fidelman N: Imaging predictors of elevated lung shunt fraction in patients being considered for yttrium-90 radioembolization. J Vasc Interv Radiol 26(10): 1472-1478, 2015. PMID: 26296737. DOI: 10.1016/j.jvir.2015.07.009

23 Joo I, Kim HC, Kim GM and Paeng JC: Imaging evaluation following ${ }^{90} \mathrm{Y}$ radioembolization of liver tumors: What radiologists should know. Korean J Radiol 19(2): 209-222, 2018. PMID: 29520178. DOI: 10.3348/kjr.2018.19.2.209

24 Lock M, Malayeri AA, Mian OY, Mayr NA, Herman JM and Lo SS: Computed tomography imaging assessment of postexternal beam radiation changes of the liver. Future Oncol 12(23): 27292739, 2016. PMID: 27576360. DOI: 10.2217/fon-2016-0165

25 Kennedy AS, Nutting C, Coldwell D, Gaiser J and Drachenberg C: Pathologic response and microdosimetry of (90) $\mathrm{Y}$ microspheres in man: review of four explanted whole livers. Int J Radiat Oncol Biol Phys 60(5): 1552-1563, 2004. PMID: 15590187. DOI: 10.1016/j.ijrobp.2004.09.004 